\title{
Unsteady flow in a 2D elastic tube with the LBGK method
}

\author{
A.G. Hoekstra*, Jos van’t Hoff, A.M. Artoli, P.M.A. Sloot \\ Section Computational Science Laboratory for Computing, Systems Architecture and Programming, Faculty of Science, \\ University of Amsterdam, Kruislaan 403, 1098 SJ Amsterdam, The Netherlands
}

Available online 20 February 2004

\begin{abstract}
We report results of unsteady, harmonic flow simulations with the lattice BGK method in two-dimensional elastic tubes. The tubes are assumed to obey a simple constitutive equation, linearly relating the diameter of the tube to the pressure difference inside and outside the tube. First, as a benchmark, we present results of steady flow in such elastic tubes, and compare the performance of three different boundary conditions for the solid wall. Next, we present results of unsteady (harmonic) flow in the elastic tube, and validate the results by comparing them with theoretical expressions for the dispersion relation of the complex speed of traveling waves in the tube. Within the range of Womersley numbers tested the agreement between the simulations and the theory is good.
\end{abstract}

(c) 2003 Elsevier B.V. All rights reserved.

Keywords: Unsteady flow; Elastic tube; Moving bounbdary; Lattice Boltzmann method

\section{Introduction}

We have a strong interest in simulating unsteady flow in complex geometry using the lattice Boltzmann BGK method (LBGK, for an introduction we refer to [15]). In particular, we focus on modeling blood flow in the large arteries of the human body. We recently showed that LBGK is in principle capable of simulating such flows within the range of Reynolds and Womersley numbers that exist in the large arteries [1]. As a proof of concept we applied LBGK to simulate flow in the lower abdominal aorta, including the bifurcation to the left and right illiac arteries (supplying the legs with blood) $[2,3]$.

\footnotetext{
* Corresponding author. Tel.: +20-525-7543; fax: +20-525-7490. E-mail addresses: alfons@science.uva.nl (A.G. Hoekstra), jvthov@icqmail.com (J. van’t Hoff), artoli@science.uva.nl (A.M. Artoli), loot@science.uva.nl (P.M.A. Sloot).

URL: http://www.science.uva.nl/research/scs.
}

In those simulations we assume that (1) blood is a Newtonian fluid and (2) that the arteries are rigid structures. The first assumption is generally acknowledged to be valid in the large arteries $[4,11,17]$. This is because of the relative high shear stress in those arteries that renders a constant viscosity of $4 \mathrm{cP}$ for whole blood. Despite this a number of groups do investigate the influence of the true non-Newtonian nature of blood on the flow profiles and shear stresses in the arteries (see, e.g. $[9,10])$. As to the second assumption, arteries as rigid structures, it is well known that, due to the pulsatile nature of blood flow and the elasticity of the arteries and surrounding tissue, during a systolic cycle the diameter of the larger arteries may vary 5-10\% (for a discussion see, e.g. [16]). However, in typical hemodynamic simulations the computational grid is obtained from Magnetic Resonance Imaging (MRI) of real arteries. Such images obtained through MRI typically have errors in the position of the artery lumen (i.e. the artery wall) of $1-8 \%$ [12]. Therefore, 
given this accuracy, the influence of elasticity of the wall can be considered a secondary effect. However, simulations do suggest that the movement of the wall may have influence on the resulting flow fields, and more important in vascular disease, on the resulting shear stress distributions on the wall (e.g. $[13,17])$. As we are currently working on developing a virtual operating theatre for vascular reconstruction $[5,6]$ the issue of wall elasticity may be of importance. We therefore aim to relax this rigid wall assumption and include elasticity of the arteries into our LBGK simulations.

In this paper we set the first steps towards including elastic walls into the LBGK model. We will study the case of two-dimensional harmonic flow in a long and slender elastic tube. This was previously done by Fang et al. [8]. They developed a tailored solid boundary condition to be used for moving walls, and studied the case of steady flow and of a harmonic flow. By comparing with theoretical expressions for steady flow they could demonstrate the validity of their approach. For the harmonic flow they solely presented simulation results. In this paper we extend the results of Fang et al. in two ways. First, we compare the performance of three different boundary conditions in the case of a steady flow in an elastic tube. Next, we perform unsteady harmonic flow simulations and compare the results with theory, especially with a dispersion relation for the complex speed of sound of the travelling waves in the tube.

\section{Theory}

The topic of unsteady flow in elastic tubes has a long history (see, e.g. [4]), with a first complete theoretical description for long and slender tubes being formulated by Womersley [18]. Since then, this theory has been refined and extended in many respects (for an overview see, e.g. [14]). However, we will use Womersley's theory to validate our simulations.

We assume a linear pressure-radius relationship:

$R=R_{0}+\frac{1}{2} \beta p$,

$p$ is the transmural pressure (i.e. the difference between the inside and outside pressure), $R_{0}$ the radius for zero transmural pressure and $\beta$ a compliance constant. This relationship is a good representation for the large arteries. Fung [4] first consider a steady flow driven by a pressure gradient. Because of the pressure drop the radius at the high-pressure end will be larger than at the low-pressure end. We assume that the tube is long and slender, that the flow is laminar and one-dimensional, and that the entry and exit effects are neglected. In that case the local flow field can be assumed to be the well-known parabolic Poiseuille profile. Using this assumption in combination with Eq. (1) and the fact that the flow rate $Q$ in every cross-section of the tube must be constant, immediately leads to the following expression: ${ }^{1}$ [4]

$3 \nu \beta Q z=[R(0)]^{4}-[R(z)]^{4}$

with $v$ is the viscosity of the fluid and $R(z)$ the radius of the tube at a distance $z$ from the inlet. By substituting the tube length $L$ in Eq. (2) we get an expression for $3 v \beta Q z$ in terms of the inlet and outlet radius. Substituting that expression back into Eq. (2) yields an explicit expression for $R(z)$ in terms of the inlet and outlet radius:

$R(z)=\left\{\left([R(L)]^{4}-[R(0)]^{4}\right) \frac{z}{L}+[R(0)]^{4}\right\}^{1 / 4}$.

Finally, by substituting Eq. (3) into Eq. (1) we obtain an expression for the pressure in the tube. Using the expression for the Reynolds number for Poiseuille flow we can now immediately derive

$R e=\frac{[R(0)]^{4}-[R(L)]^{4}}{2 \beta L v^{2}}$.

The analytical solution for time-dependent flow in an elastic tube was first described by Womersley [18]. Here we just provide an outline of his theory. For all details we refer to the original paper. The theory assumes that the non-linear terms in the Navier-Stokes equations may be ignored (i.e. small Reynolds number flows). The tube is assumed to be a thin-walled elastic tube with radius $R$, wall thickness $h$, density of the tube wall $\rho$. Moreover, the density of the fluid is $\rho_{0}$. The equations for the displacement of the wall and the fluid motion are coupled through a set a boundary conditions at the inner wall of the tube. Introducing a speed of sound $c$ and looking at the type of motion

\footnotetext{
${ }^{1}$ Note that Eq. (2) differs from the expression in Ref. [4] because we consider two-dimensional flows here.
} 
in which $u / c, v / c$, and $\omega R / c$ are small (with $u$ and $v$ the longitudinal and radial components of the fluid velocity, and $\omega$ the angular frequency of the driving harmonic pressure gradient), we can write

$p=p_{1} \exp \left[\mathrm{i} \omega\left(t-\frac{z}{c}\right)\right]$,

$u=u_{1} \exp \left[\mathrm{i} \omega\left(t-\frac{z}{c}\right)\right]$,

$v=v_{1} \exp \left[\mathrm{i} \omega\left(t-\frac{z}{c}\right)\right]$.

Next, the equations of motion can be solved and in combination with the boundary conditions four homogeneous linear equations in four unknowns are obtained. A non-trivial solution is only possible by requiring that the determinant of this system of equations is zero, thus resulting in a dispersion relation which will determine the wave velocity $c$ in terms of the elastic properties of the tube and $\alpha$, the non-dimensional Womersley number $(\alpha=R \sqrt{\omega / v})$. The final dispersion relation reads

$$
\begin{aligned}
& \left(1-\sigma^{2}\right)(1-F) x^{2}-x\left[2+k(1-F)+F\left(\frac{1}{2}-2 \sigma\right)\right] \\
& \quad+F+2 k=0
\end{aligned}
$$

where

$F=2 \frac{J_{1}\left(\alpha \mathrm{i}^{3 / 2}\right)}{\alpha \mathrm{i}^{3 / 2} J_{0}\left(\alpha \mathrm{i}^{3 / 2}\right)}$,

$k=\frac{h p}{R \rho_{0}}$,

$x=\frac{2}{\left(1-\sigma^{2}\right)} \frac{c_{0}^{2}}{c^{2}}$

with $\sigma$ Poisson's ratio of the elastic material of the tube, $J_{0}$ and $J_{1}$ the zeroth- and first-order Bessel functions, and $c_{0}$ the speed of sound in the 'empty' tube. By solving Eq. (8) we find a, in general complex valued expression for $x$. If we now write

$\frac{c_{0}}{c}=\sqrt{\left(1-\sigma^{2}\right) \frac{x}{2}}=X-\mathrm{i} Y$,

and substitute this into Eq. (5) we immediately recognize that the wave velocity $c_{1}$ of the pressure waves in the tube becomes

$c_{1}=\frac{c_{0}}{X}$ and that the wave after travelling over a distance of one wavelength is attenuated by a factor $\gamma$

$\gamma=\exp \left[-2 \pi \frac{Y}{X}\right]$.

\section{Results}

\subsection{Description of the simulations}

For the fluid flow simulations we apply the D2Q9 Lattice BGK model [15]. For the solid walls we have tested three different boundary conditions. The Velocity Boundary Condition (VBC) by Zou and He [19], the recently proposed boundary condition by Bouzidi et al. [7] and another recently proposed boundary condition by Fang et al. [8]. The last one was specifically developed with moving boundaries in mind. At the inlet and outlet we use pressure boundary conditions. For steady flows a constant pressure difference is prescribed. For the unsteady flow the density at the inlet was set to $1+0.001 \cos (2 \pi t / T)$ and at the outlet to 1. Here, $t$ denotes time and $T$ the period of oscillation (both measured in units of $\Delta t=1$ ). As the solid walls move during the simulation, it may happen that fluid nodes turn into boundary nodes and boundary nodes into solid nodes, or the other way around. If a boundary node turns into a fluid node we need some prescription to assign values to the yet unknown distribution function on the new fluid nodes. For this we use the method described by Fang et al. [8], where the unknown distribution functions are assigned through second-order extrapolation in the directions that pointed towards fluid nodes in the previous time step. The coupling between the fluid and the elastic wall is through Eq. (1). We first start a simulation with a fixed wall and allow the flow field to reach a steady state. Next, the wall is released. In every cross-section of the tube the pressure is measured, and the radius is instantaneously adapted to the value given by Eq. (1). Next, an LBGK iteration is performed, the pressure is again measured, etc. This cycle is iterated until the tube has settled (in case of steady flow) or performs steady harmonic oscillations (in case of the harmonic flow). Fig. 1 shows an example of this settling of the tube wall for both steady and periodic flows. For harmonic flow in tubes with a smaller inlet diameter the convergence is faster (data not shown). 

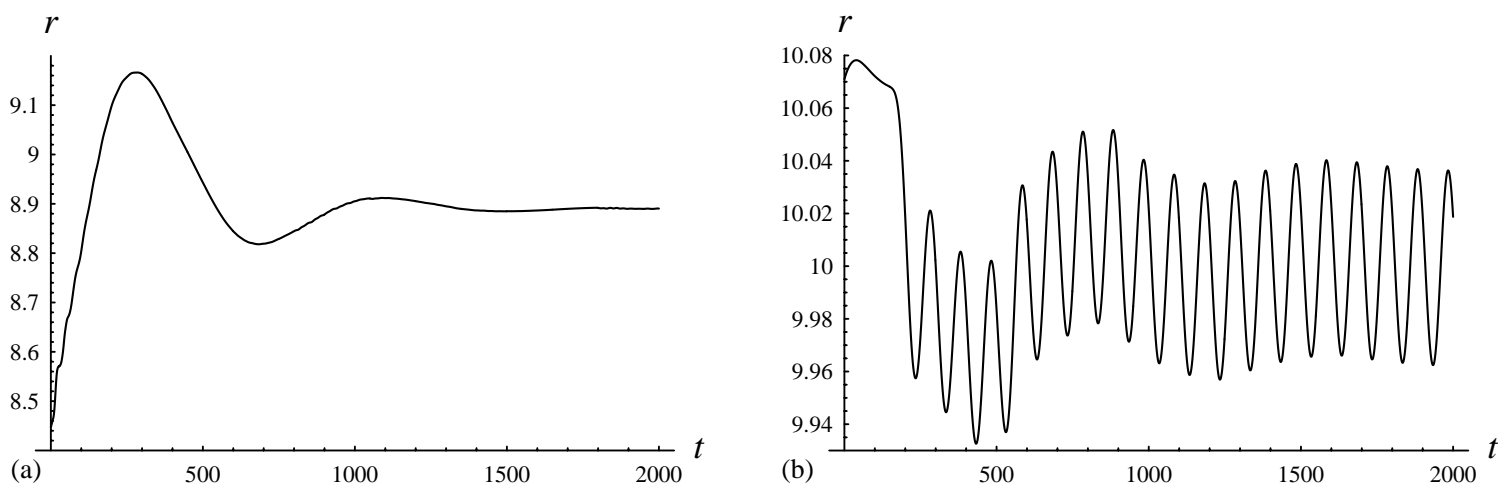

Fig. 1. Settling of the radius (vertical axis) at $z=L / 2$ in the 2000 time steps (horizontal axis) after the walls are released. Left panel: steady flow on a $20 \times 100$ lattice $(R e=1)$; right panel: harmonic flow on a $10 \times 100$ lattice with $T=100$.

The reason for not starting the simulation immediately with elastic walls is that the stability of the simulations enhances significantly if the flow is first allowed to settle in the uniform rigid tube (data not shown).

\subsection{Steady flow}

We have performed a set of simulations where the length of the tube was 600 , the inlet diameter was set to 30 and the outlet diameter to 21 . The Reynolds number was set to 1 and 10, respectively. First we show, in Fig. 2, the resulting diameter as a function of position in the tube for the three boundary conditions. The theoretical curve (Eq. (3)) is shown as well. For other choices of diameters the results are comparable. We calculate an average error defined by

$\epsilon=\frac{\Sigma\left|R_{\mathrm{T}}(z)-R_{\mathrm{S}}(z)\right|}{\Sigma\left|R_{\mathrm{T}}(z)\right|}$,
Table 1

Relative errors in the shape of an elastic tube at several Reynolds numbers

\begin{tabular}{lll}
\hline Boundary condition & $R e=1$ & $R e=10$ \\
\hline Fang & 0.0006 & 0.0014 \\
Bouzidi & 0.0063 & 0.0059 \\
Zou & 0.0006 & 0.0013 \\
\hline
\end{tabular}

where the subscript $\mathrm{T}$ denotes the theory and $\mathrm{S}$ the simulation. Table 1 shows the resulting errors.

We have also measured the error in the resulting velocity fields (using the same error definition as in Eq. (15)), as a function of the grid spacing. The results are presented in Fig. 3. The error behavior is first order in the grid spacing (to be precise, for $R e=1$ the error goes as $\Delta x^{1.4}$ for all three boundary conditions, and for $R e=10$ as $\Delta x^{1.4}$ for the Bouzidi boundary condition and as $\Delta x^{1.1}$ for the other two).
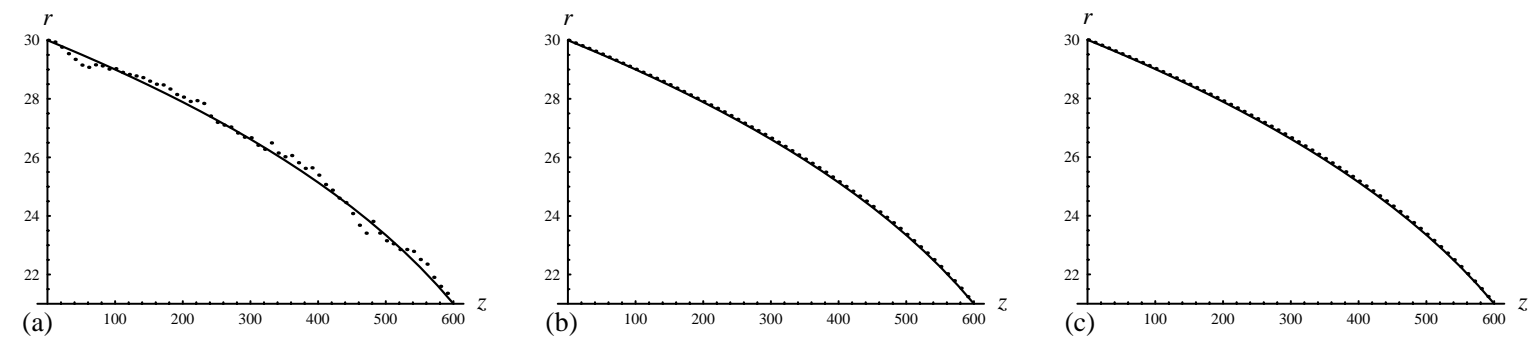

Fig. 2. Theoretical shape of the upper wall of an elastic tube due to steady flow compared to the simulated results of the Bouzidi (a), Zou (b) and Fang boundary (c) at $R e=10$. 

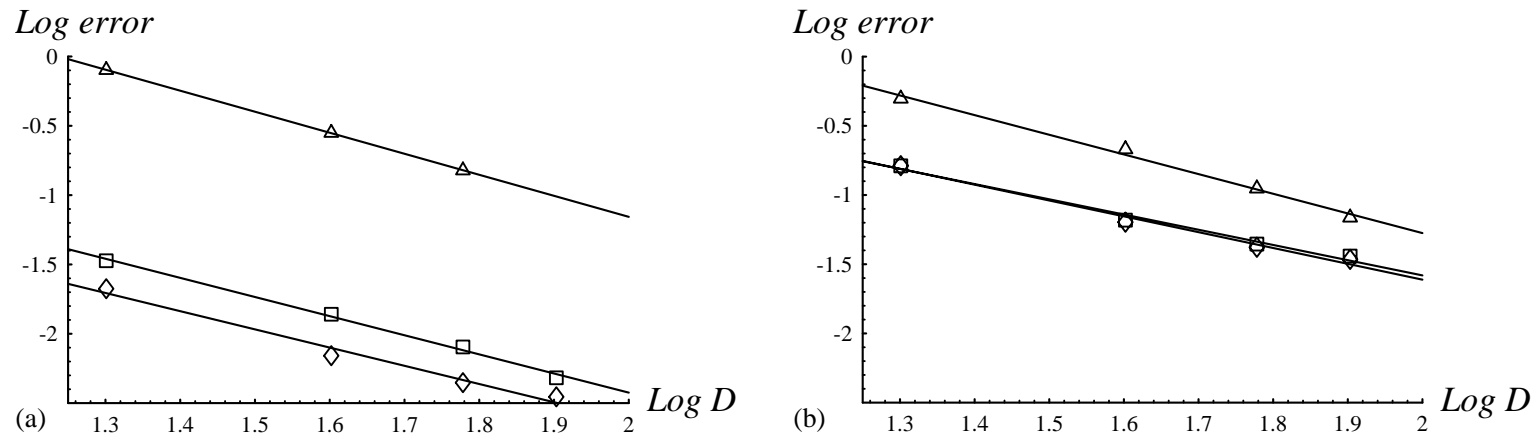

Fig. 3. Relative errors in velocity profiles as a function of the grid spacing for the Fang (diamond), Bouzidi (box) and Zou (triangle) boundary conditions at Reynolds numbers 1 (left) and 10 (right). Solid lines are the linear fits.

\subsection{Unsteady flow}

For the unsteady flow simulations we only applied the Fang boundary. In Fig. 4 we show the propagating pressure wave that is induced in the elastic tube. Comparing the results of the simulations to the dispersion relation in Eq. (6) is done by analyzing these pressure waves. The only parameter that was changed for different simulations is the radius of the tube under zero transmural pressure. With the radius, the Womersley number also changes. This has an effect on the attenuation of the amplitude of the pressure wave (through the function $F$, see Eq. (7)). For large $\alpha$ the amplitude decreases less per wavelength than for small $\alpha$.

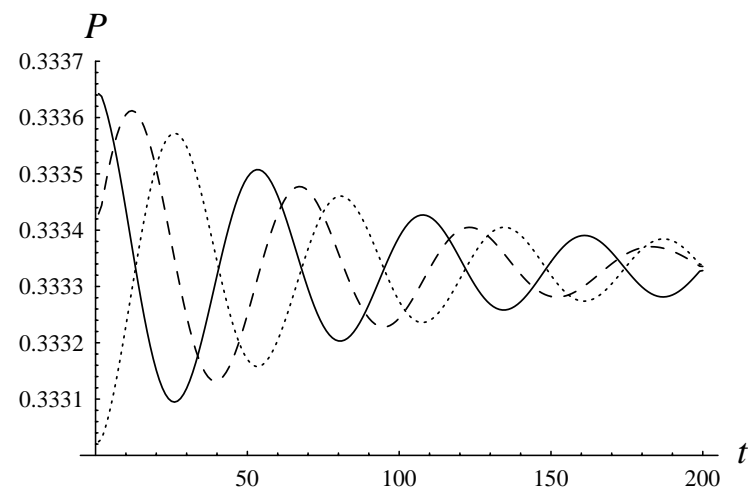

Fig. 4. Propagation of the pressure wave. The pressure distributions at $t=20 T$ (solid line), 20.25T (dashed line) and 20.5T (dotted line) are shown for a tube of length 200 and $R=10(T=100$ and $\alpha=6.14)$.
If we put the Poisson ratio and the thickness of the wall to zero in Eq. (6), which is the case for our simulations, we find for the attenuation constant $\gamma$ the following theoretical values: at $\alpha=3.07$ an attenuation constant of 0.102 ; if we then increase the radius by a factor 2 , which increases $\alpha$ to 6.14 , we find an attenuation constant of 0.409 . This means the amplitude of the pressure wave drops faster along the length of the tube for lower Womersley number. The attenuation constant $\gamma$ is obtained from the simulations by fitting the simulated pressure waves with the theoretical expressions. The results, for a range of Womersley parameters, are shown in Fig. 5 and Table 2.

For larger Womersley numbers the agreement between simulation and theory is perfect. However, for the small $\alpha$-values we observe a large difference.

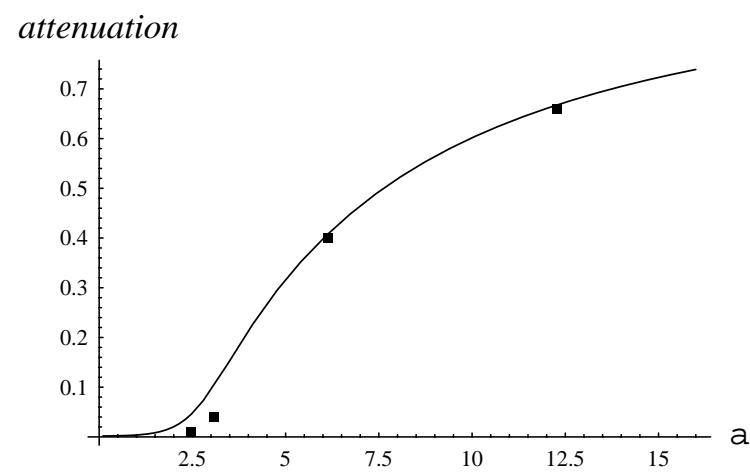

(a)

Fig. 5. Simulated (box) and theoretical (line) attenuation constants as a function of the Womersley number. The line represents the theory $(\sigma=0, k=0)$. 
Table 2

Numerical values of the theoretical and simulated attenuation constants presented in Fig. 5

\begin{tabular}{rll}
\hline$\alpha$ & $\begin{array}{l}\text { Theoretical } \\
\text { attenuation constant }\end{array}$ & $\begin{array}{l}\text { Simulated attenuation } \\
\text { constant }\end{array}$ \\
\hline 2.46 & 0.04 & 0.01 \\
3.07 & 0.10 & 0.04 \\
6.14 & 0.41 & 0.40 \\
12.28 & 0.67 & 0.66 \\
\hline
\end{tabular}

Table 3

Numerical values of the theoretical and simulated attenuation constants presented in Fig. 5

\begin{tabular}{lrll}
\hline$\alpha_{1}$ & \multicolumn{2}{l}{$\lambda \alpha_{2}$} & \multicolumn{2}{l}{$\lambda\left(\alpha_{1}\right) / \lambda\left(\alpha_{2}\right)$} & \\
\cline { 3 - 4 } & & Theory & Simulated \\
\hline 2.46 & 3.07 & 0.94 & $0.98 \pm 0.07$ \\
2.46 & 6.14 & 0.86 & $0.95 \pm 0.07$ \\
2.46 & 12.28 & 0.82 & $0.93 \pm 0.07$ \\
3.07 & 6.14 & 0.92 & $0.96 \pm 0.07$ \\
3.07 & 12.28 & 0.88 & $0.95 \pm 0.07$ \\
6.14 & 12.28 & 0.95 & $0.98 \pm 0.07$ \\
\hline
\end{tabular}

This discrepancy may be attributed to discretization errors. For instance, for $\alpha=2.46$ the radius of the inlet was only 5 grid points. We performed another simulation for the same $\alpha$ but now with a radius of 20 grid points (and a tube length of 3500 and $T=$ 2500 , thus dramatically increasing execution times to $80 \mathrm{~h}$ on a $300 \mathrm{MHz}$ Sun workstation). After fitting we now obtained $\gamma=0.031$. This result is much closer to the theory, but still the error is relatively large.

The influence from the compliance constant (i.e. the elasticity) of the wall on the pressure waves comes only through $c_{0}$, the wave velocity for a non-viscous fluid. ${ }^{2}$ In other words, we expect that the attenuation is not influenced by the compliance constant. To check this we ran a simulation for $\alpha=6.14$ with a twice as large compliance constant. In that case we found $\gamma=0.43$.

We have also measured the wavelength of the pressure waves $\lambda=c_{0} T / X$. Unfortunately, because of our specific simulation setup a theoretical expression for $c_{0}$ was not immediately obvious. Therefore, we have

\footnotetext{
${ }^{2}$ And of course also through the Poisson's Ratio, but here we have taken $\sigma=0$.
}

looked at ratios of measured wavelengths at different Womersley numbers, which only depend on $X(\alpha)$. The results are shown in Table 3.

\section{Discussion}

We presented a first set of results of LBGK simulations with elastic walls. From our steady flow benchmarks we observe that the Fang and Zou boundary have comparable error behavior. Notice that in combination with moving walls the error in the velocity is first order in the grid spacing. The Bouzidi boundary also is first order, but has a much larger value of the errors. For non-moving walls this boundary condition is very good (see, e.g. [2]). However, in combination with elastic walls our results suggest that the details of the interpolations result in a wrong density (and therefore pressure) field close to the wall. This has an immediate effect on the location of the walls, see Eq. (1) and the resulting velocity fields. In the simulations for unsteady flow we have used the Fang boundary. For a rigid tube we were able to perfectly reproduce Womersley flow with this boundary condition (data not shown). For the elastic wall the results are very promising. We reproduce the attenuated pressure waves as predicted by theory. Moreover, over a range of Womersley numbers we have demonstrated a good agreement between the theoretical dispersion relation for the complex wave velocity and our simulations. The relative large errors for small Womersley number need further investigation, but the suggested discretization effect may play an important role. The elastic wall in our simulations was highly simplified: it was massless and had a zero thickness. This is not very realistic, and as a next step we will consider tubes with a thin wall and non-zero mass. This means that we will also have to integrate the equations of motion of the wall. We will consider other constitutive equations for the wall, such as a linear material described by a Young's modulus and a Poisson ratio. In this case the $c_{0}$ is given by the Moens-Korteweg formula [4]. This will allow a more extensive validation of our simulations, by comparison with Eq. (6) over a large range of parameter values. Moreover, we will also extend the simulations to three dimensions, and consider more realistic non-symmetrical cases. 


\section{References}

[1] A.M. Artoli, A.G. Hoekstra, P.M.A. Sloot, 3D pulsatile flow in the lattice Boltzmann BGK method, Int. J. Mod. Phys. C 13 (2002) 1119-1134.

[2] A.M. Artoli, A.G. Hoekstra, P.M.A. Sloot, Simulation of a systolic cycle in a realistic artery with the lattice Boltzmann BGK method, Int. J. Mod. Phys. B 17 (2003) 95-98.

[3] A.M. Artoli, A.G. Hoekstra, P.M.A. Sloot, Mesoscopic simulations of flow in the human abdominal aorta, J. Biomech., accepted for publication, 2004.

[4] Y.C. Fung, Biomechanics, Circulation, Springer, New York, 1984.

[5] R.G. Belleman, P.M.A. Sloot, The design of dynamic exploration environments for computational steering simulations, in: M. Bubak, J. Moscinski, M. Noga (Eds.), Proceedings of the SGI Users' Conference 2000, 2000, pp. 57-74. ISBN 83-902363-9-7.

[6] R.G. Belleman, P.M.A. Sloot, Simulated vascular reconstruction in a virtual operating theatre, in: H.U. Lemke, M.W. Vannier, K. Inamura, A.G. Farman, K. Doi (Eds.), Computer Assisted Radiology and Surgery, Excerpta Medica, Int. Cong. Ser. 1230 (2001) 938-944.

[7] M. Bouzidi, M. Firdaouss, P. Lallemand, Momentum transfer of a lattice Boltzmann fluid with boundaries, Phys. Fluids 13 (2001) 3452-3459.

[8] H. Fang, Z. Wang, Z. Lin, M. Liu, Lattice Boltzmann method for simulating the viscous flow in large distensible arteries, Phys. Rev. E 65 (2002) 051925.

[9] F.J.H. Gijsen, E. Allanic, F.N. van de Vosse, J.D. Janssen, The influence of the non-Newtonian properties of blood on the flow in large arteries: unsteady flow in a 900 curved tube, J. Biomech. 32 (1999) 705-713.

[10] F.J.H. Gijsen, F.N. van de Vosse, J.D. Janssen, The influence of the non-Newtonian properties of blood on the flow in large arteries: steady flow in a carotid bifurcation model, J. Biomech. 32 (1999) 601-608.

[11] D.N. Ku, Blood flow in arteries, Annu. Rev. Fluid Mech. 29 (1997) 399-434.

[12] J.A. Moore, D.A. Steinman, C.R. Ethier, Computational blood flow modeling: errors associated with reconstructing finite element models from magnetic resonance images, J. Biomech. 31 (1998) 179-184.

[13] K. Perktold, G. Rappitsch, Computer simulations of local blood flow and vessel mechanics in a compliant carotid artery bifurcation model, J. Biomech. 28 (1995) 845-856.

[14] B.B. Sramek, J. Valenta, F. Klimes (Eds.), Biomechanics of the Cardio-Vascular System, Czech Technical University Press, Prague, 1995.

[15] S. Succi, The Lattice Boltzmann Equation for Fluid Dynamics and Beyond, Oxford University Press, Oxford, 2001.

[16] C.A. Taylor, T.J.R. Hughes, C.K. Zarins, Finite element modeling of three-dimensional pulsatile flow in the abdominal aorta: relevance to atherosclerosis, Ann. Biomed. Eng. 26 (1998) 975-987.
[17] D.A. Vorp, D.A. Steinman, C.R. Ethier, Computational modeling of arterial biomechanics, IEEE Comput. Sci. Eng. 3 (2001) 51-64.

[18] J.R. Womersley, Oscillatory motion of a viscous liquid in a thin-walled elastic tube. I. The linear approximation for long waves, Phil. Mag. 46 (1955) 199-221.

[19] Q. Zou, X. He, On pressure and velocity boundary conditions for the lattice Boltzmann BGK model, Phys. Fluids 9 (1997) 1591.

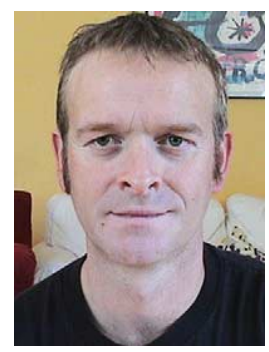

Dr. A.G. Hoekstra holds a $\mathrm{PhD}$ in computational science from the University of Amsterdam (1994) in the field of parallel and distributed computing. After 2 years of PostDoc research on parallel hierarchical algorithms for many body problems he became assistant professor at the University of Amsterdam, working on parallel and distributed computing and particle based modeling and simulation. Currently he is an associate professor at the University of Amsterdam, with a strong research interest in scientific computing on the grid and lattice Boltzmann modeling and simulation. He has organized International Conferences in the field of high performance computing and computational science. He has published over a 100 peer reviewed research papers.

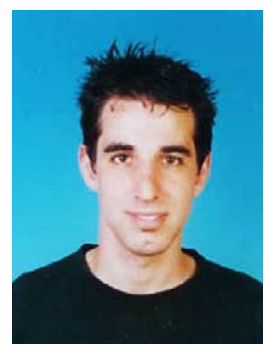

Jos van't Hoff holds a Master's degree in computational physics from the University of Amsterdam. His interest is in mesoscopic models for fluids and fluid-solid interactions.

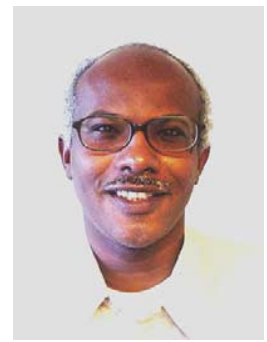

Dr. A.M. Artoli graduated in physics at Assiut University, Egypt, obtained his MSc in physics at the University of Khartoum, Sudan and received his $\mathrm{PhD}$ in the Computational Science Section, Department of Computer Science at the University of Amsterdam in 2003 on mesoscopic computational haemodynamics. He is an assistant professor at the School of Applied Physics, Faculty of Science and Technology, Al Neelain University, Khartoum, Sudan. His current research interests include biofluid computational mechanics. 


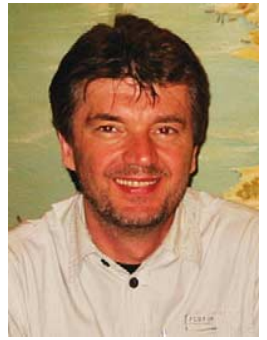

Prof. Dr. P.M.A. Sloot (http://www. science.uva.nl/ $\sim$ sloot $/$ ), graduated in physics and chemistry at the University of Amsterdam and worked for 6 years in The Netherlands Cancer Institute as a research assistant. $\mathrm{He}$ received his $\mathrm{PhD}$ in computer science in 1988 at the UvA, was visiting professor in various international universities among which Bandung (Indonesia), St. Petersburg (Russia), and the Santa Fe Institute on complex systems (USA). From 1996 to 2001 he was granted a professorship as the NNV distinguished chair on computational physics. Since 2002 he is full professor computational science at the UvA. He has initiated over eight EU research and development projects in ICT, is co-leading the EU Cross-Grid project and one of the board members of the EU Health-Grid Initiative. Over the last 10 years he has been in a large number of national and international committees on high performance computing. With Jack Dongarra he is responsible for the International Conferences on Computational Science series. He has been elected by the European Science Foundation as the chair of the Problem Solving Environment Programme. He has published over 200 peer reviewed papers and books on various topics in computer science and guided over $10 \mathrm{PhD}$ theses. 